\title{
Magnetic instability in a sheared azimuthal flow
}

\author{
A. P. Willis and C. F. Barenghi \\ Department of Mathematics, University of Newcastle, Newcastle upon Tyne, NE2 7RU, UK \\ Received 8 January 2002 / Accepted 3 April 2002

\begin{abstract}
We study the magneto-rotational instability of an incompressible flow which rotates with angular velocity $\Omega(r)=a+b / r^{2}$ where $r$ is the radius and $a$ and $b$ are constants. We find that an applied magnetic field destabilises the flow, in agreement with the results of Rüdiger \& Zhang (2001). We extend the investigation in the region of parameter space which is Rayleigh stable. We also study the instability at values of magnetic Prandtl number which are much larger and smaller than Rüdiger \& Zhang. Large magnetic Prandtl numbers are motivated by their possible relevance in the central region of galaxies (Kulsrud \& Anderson 1992). In this regime we find that increasing the magnetic Prandtl number greatly enhances the instability; the stability boundary drops below the Rayleigh line and tends toward the solid body rotation line. Very small magnetic Prandtl numbers are motivated by the current MHD dynamo experiments performed using liquid sodium and gallium. Our finding in this regime confirms Rüdiger \& Zhang's conjecture that the linear magneto-rotational instability and the nonlinear hydrodynamical instability (Richard \& Zahn 1999) take place at Reynolds numbers of the same order of magnitude.
\end{abstract}

Key words. accretion discs - instabilities - magnetohydrodynamics - turbulence

\section{Motivation}

It is thought that turbulence in accretion discs arises from a magneto-rotational instability (MRI), where a magnetic field destabilises a rotating velocity field which decreases outwardly. This instability was discovered by Velikhov (1959) and Chandrasekhar (1961) when studying the motion of a fluid between two concentric cylinders (Taylor-Couette flow). It was only years later, when it was realized that Couette flow can be interpreted as model of Keplerian flow, that the implications for astrophysics were fully appreciated (Balbus \& Hawley 1991). There have been many studies of the MRI recently, from numerical simulations of accretion discs (Brandenburg et al. 1995) to nonlinear calculations in spherical geometry (Drecker et al. 2000). In particular, in a recent paper Rüdiger \& Zhang (2001) analysed the linear stability of hydromagnetic Couette flow and showed that, if the magnetic Prandtl number is less than unity, azimuthal Couette flow is more easily destabilised with a magnetic field than without. They found that the instability extends into the region of parameter space which is Rayleigh-stable without a magnetic field, which is important since Keplerian rotation is Rayleigh-stable. Rüdiger \& Zhang were also able to study the MRI instability at magnetic Prandtl numbers $P m$ as small as 0.001 , towards the limit relevant

Send offprint requests to: C. F. Barenghi, e-mail: c.f.barenghi@newcastle.ac.uk to liquid sodium and gallium $\left(P m \approx 10^{-5}\right)$ which are used in current MHD dynamo experiments (Tilgner 2000).

The aim of this paper to is to extend the investigation of Rüdiger \& Zhang (2001). We explore the instability as a function of the speed of the outer cylinder in the Rayleigh-stable region, which is the parameter space of astrophysical interest (Rüdiger \& Zhang considered only one nonzero ratio of outer to inner cylinder's rotation). We also determine the effect of changing the magnetic Prandtl number, extending the range studied by Rüdiger \& Zhang.

\section{Model}

We consider an incompressible fluid contained between two coaxial cylinders of inner radius $R_{1}$ and outer radius $R_{2}$ which rotate at prescribed angular velocities $\Omega_{1}$ and $\Omega_{2}$. We use cylindrical coordinates $(r, \phi, z)$ and assume that a uniform magnetic field of strength $B_{0}$ is applied in the axial $z$ direction. At small angular velocities the flow is purely azimuthal (circular-Couette flow) and has magnitude $V_{0}(r)=a r+b / r$ which corresponds to the rotation law,

$\Omega(r)=\frac{V_{0}}{r}=a+\frac{b}{r^{2}}$.

The constants $a$ and $b$ are determined by the no-slip boundary conditions at the cylinder's walls, so we have,

$a=\Omega_{1} \frac{\left(R_{1}^{2} / R_{2}^{2}-\Omega_{2} / \Omega_{1}\right)}{\left(1-R_{1}^{2} / R_{2}^{2}\right)}, \quad b=\Omega_{1} R_{1}^{2} \frac{\left(1-\Omega_{2} / \Omega_{1}\right)}{\left(1-R_{1}^{2} / R_{2}^{2}\right)}$. 
At sufficiently high angular velocities Couette flow becomes unstable. The resulting velocity and magnetic fields $\boldsymbol{V}(r, \phi, z, t)$ and $\boldsymbol{B}(r, \phi, z, t)$ are determined by the MHD equations which we write in dimensionless form as,

$$
\begin{aligned}
& \frac{\partial \boldsymbol{V}}{\partial t}+(\boldsymbol{V} \cdot \nabla) \boldsymbol{V}=-\nabla p+\nabla^{2} \boldsymbol{V}+\frac{Q}{P m}(\nabla \wedge \boldsymbol{B}) \wedge \boldsymbol{B} \\
& \frac{\partial \boldsymbol{B}}{\partial t}=\frac{1}{P m} \nabla^{2} \boldsymbol{B}+\nabla \wedge(\boldsymbol{V} \wedge \boldsymbol{B}) \\
& \boldsymbol{\nabla} \cdot \boldsymbol{B}=0, \quad \boldsymbol{\nabla} \cdot \boldsymbol{V}=0
\end{aligned}
$$

where $p$ is the pressure. In writing Eqs. (3) and (4) we used $\delta=R_{2}-R_{1}$ as unit of length, $\delta^{2} / \nu$ as unit of time and $B_{0}$ as unit of magnetic field, where $\nu$ is the kinematic viscosity. The governing dimensionless parameters of the problem are the Reynolds numbers $R e_{1}, R e_{2}$, radius ratio $\eta$, and rotation ratio $\mu$,

$R e_{1}=\frac{R_{1} \Omega_{1} \delta}{\nu}, \quad R e_{2}=\frac{R_{2} \Omega_{2} \delta}{\nu}, \quad \eta=\frac{R_{1}}{R_{2}}, \quad \mu=\frac{\Omega_{2}}{\Omega_{1}}$,

together with Chandrasekhar's number $Q$ and the magnetic Prandtl number $P m$,

$Q=\frac{B_{0}^{2} \delta^{2} \sigma}{\rho \nu}, \quad P m=\frac{\nu}{\lambda}$,

where $\rho$ is the density, $\sigma$ is the electrical conductivity, $\lambda=1 /\left(\sigma \mu_{0}\right)$ is the magnetic diffusivity and $\mu_{0}$ is the permeability.

We solve the MHD equations by direct time stepping from a small seeding initial condition and determine the stability of the Couette solution in different regions of parameter space. Our numerical method for 3D nonlinear flow is detailed in Willis \& Barenghi (2002). For this linear study it suffices to say that the formulation is based on representing $\boldsymbol{V}$ and $\boldsymbol{B}$ with suitable potentials which are spectrally expanded over Fourier modes exp $[\mathrm{i} m \phi+\mathrm{i} \alpha z]$ in the azimuthal and axial directions and over Chebyshev polynomials in the radial direction. The axial wavelength of the disturbance is $2 \pi / \alpha$. We assume no-slip boundary conditions for $\boldsymbol{V}$, and electrically insulating boundaries conditions for $\boldsymbol{B}$,

$\frac{\partial B_{r}}{\partial z}=\frac{B_{z}}{\mathcal{B}_{m}} \frac{\partial \mathcal{B}_{m}}{\partial r}, \quad \frac{1}{r} \frac{\partial B_{z}}{\partial \phi}=\frac{\partial B_{\phi}}{\partial z}$,

where $\mathcal{B}_{m}(r)$ denotes the modified Bessel functions, $I_{m}(\alpha r)$ on $R_{1}$, and $K_{m}(\alpha r)$ on $R_{2}$. The time stepping is based on a combination of second order accurate CrankNicolson and Adams-Bashforth methods. The resulting computer code was tested against published results with and without magnetic field (Chandrasekhar 1961; Roberts 1964; Marcus 1984; Jones 1985; Barenghi 1991).

\section{Results}

It is well known that, in the absence of magnetic field, inviscid Couette flow is linearly stable provided that the celebrated Rayleigh criterion $\left(\mu>\eta^{2}\right)$ is satisfied.

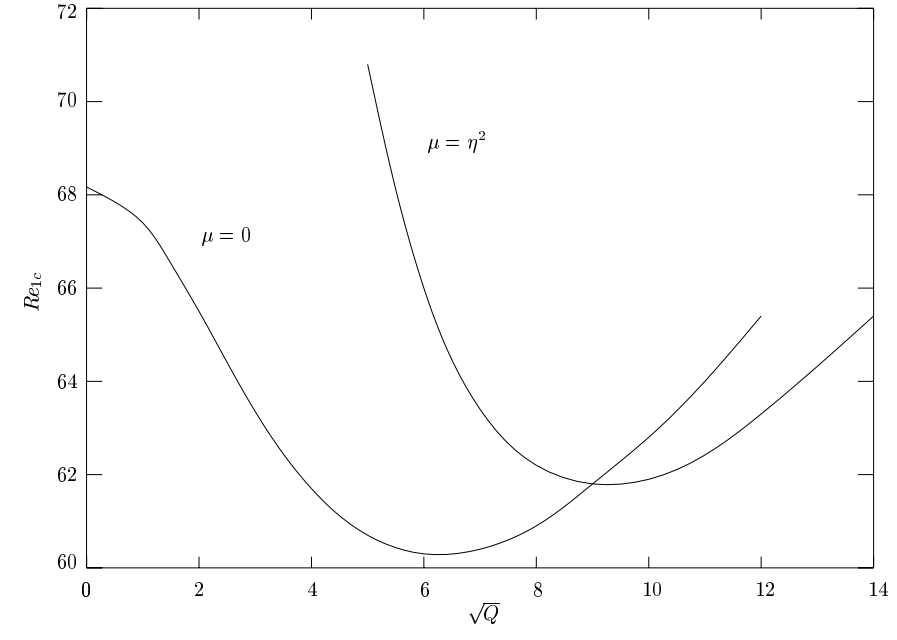

Fig. 1. $\eta=0.5, P m=1$. Critical Reynolds number of the inner cylinder $\left(R e_{1 \mathrm{c}}\right)$ versus applied magnetic field $(\sqrt{Q})$; two cases are presented: outer cylinder fixed $(\mu=0)$ and on the Rayleigh line $\left(\mu=\eta^{2}\right)$.

Figure 1 shows the result of our calculations for the stability of dissipative Couette flow to axisymmetric perturbations, for radius ratio $\eta=0.5$ and magnetic Prandtl number $P m=1$, as a function of the applied magnetic field. We plot the result in terms of $\sqrt{Q}$ rather than $Q$ in order to make direct comparison with the work of Rüdiger \& Zhang (2001). The first curve refers to the case in which the outer cylinder is fixed, $\mu=0$, which is the most studied case in the fluid dynamics literature. It is apparent that the presence of a magnetic field makes the flow more unstable. The critical Reynolds number, which is $R e_{1 \mathrm{c}}=68.2$ for $Q=0$, decreases with increasing $Q$ and has a minimum at $Q=39$. The most unstable mode is $m=0$ over the range for $Q$ in Fig. 1 . From here we consider axisymmetric disturbances only. The critical axial wavenumber $\alpha$ decreases significantly from 3.1 to 1.7 over the range, and varies like $1 / \sqrt{Q}$ thereafter. This stiffening eventually restabilises the flow and $R e_{1 \mathrm{c}}$ increases like $\sqrt{Q}$ for strong fields.

The initial destabilisation is consistent with the finding of Rüdiger \& Zhang (2001); the small difference between their $R e_{1 \mathrm{c}}$ and ours is certainly due to the different boundary conditions for $\boldsymbol{B}$ (they assumed pseudo-vacuum conditions and we assume insulating conditions). The second curve of Fig. 1 refers to the case $\mu=\eta^{2}$ (the Rayleigh line), which separates stable and unstable regions in the absence of a magnetic field. The curve well illustrates the striking destabilising effect of the magnetic field. Figure 2 shows stability boundaries in the $R e_{1}$ vs. $R e_{2}$ plane for different values of the imposed magnetic field. The axial wavenumber $\alpha$ does not vary a great deal along the boundaries, its dependence being principally determined by the strength of the field $Q$.

It is apparent that even a small value of $Q$ is enough to make the boundary cross the Rayleigh line (the upper dotted curve in the figure). 


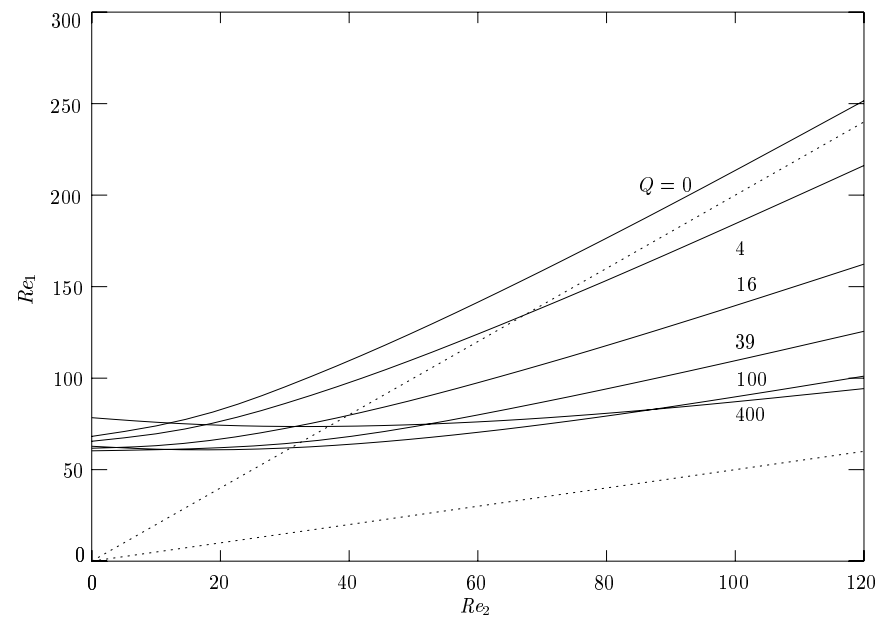

Fig. 2. $\eta=0.5, P m=1$. Critical Reynolds number of the inner cylinder $\left(R e_{1 \mathrm{c}}\right)$ versus Reynolds number of the outer cylinder $\left(R e_{2}\right)$ at different values of applied magnetic field $(Q)$. The upper dotted line is the Rayleigh criterion. The lower dotted line is solid-body rotation.

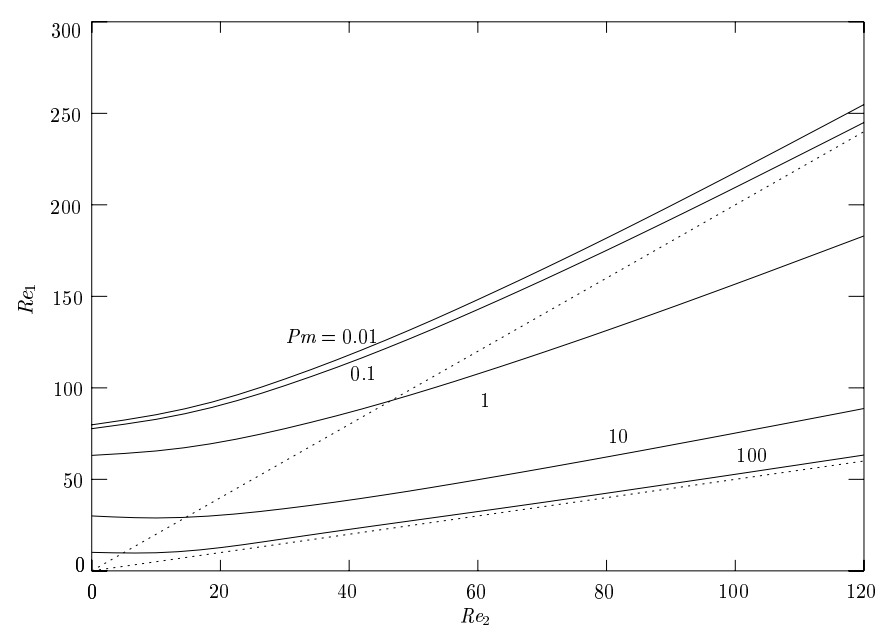

Fig. 3. $\eta=0.5, Q=10$. Critical Reynolds number of the inner cylinder $\left(R e_{1 \mathrm{c}}\right)$ versus Reynolds number of the outer cylinder $\left(R e_{2}\right)$ at different values of magnetic Prandtl number $(P m)$. The upper dotted line is the Rayleigh criterion. The lower dotted line is solid-body rotation.

The destabilising effect of the magnetic field is so large that the stability boundary drops toward the region of solid body rotation $\left(\Omega_{1}=\Omega_{2}\right.$ or $\left.\mu=1\right)$, which is the lower dotted line. However, if the applied field is strong enough the flow can be restabilised, in accordance with the results of Fig. 1. The destabilisation becomes more important the larger the magnetic Prandtl number Pm. Figure 3 shows results for a moderate value of applied magnetic field, $Q=$ 10, at increasing values of $P m$. It is apparent that the stability boundary drops much below the Rayleigh line $\mu=\eta^{2}$ and, for large enough $P m$, becomes asymptotic to the solid body rotation line $\mu=1$.

Finally, in Fig. 4 we find that on the Rayleigh line the stability follows a power law,

$R e_{1 \mathrm{c}} \propto P m^{-\beta}, \quad \beta=-0.500 \pm 0.002$,

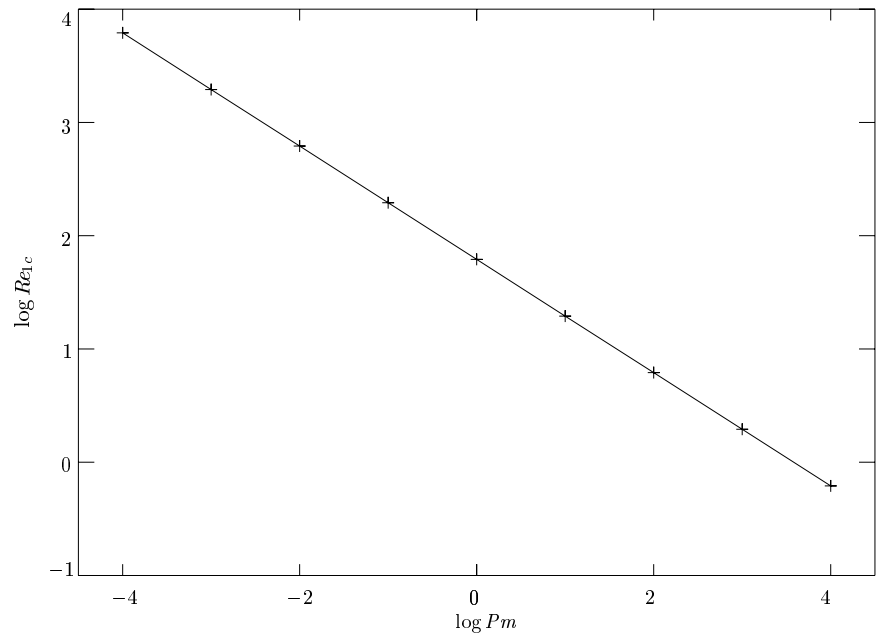

Fig. 4. $\eta=0.5$. Critical Reynolds number of the inner cylinder $\left(R e_{1 \mathrm{c}}\right)$ versus magnetic Prandtl number $(P m)$ on the Rayleigh line, $\mu=\eta^{2}$.

over a surprising range of $P m$. The error in $\beta$ is based on a fit through all points calculated. For $\eta=0.5$ the minimum $R e_{1 \mathrm{c}}$ occurs at $Q=86$ and $\alpha=2.1$. To verify this result we use the WKB analysis of Ji et al. (2001). The local dispersion relation they find for the Taylor-Couette flow is identical to the one derived for accretion discs in the incompressible limit (Sano \& Miyama 1999). From the dispersion relation the necessary and sufficient condition for stability to axisymmetric perturbations is,

$\left(P m+S^{2}\right)^{2}\left(1+\epsilon^{2}\right)+2 \zeta R m^{2}-2(2-\zeta) R m^{2} S^{2} \geq 0$,

where $S$ is the ratio of diffusive to Alfvén timescales, $R m$ is the magnetic Reynolds number, and $\zeta=1 /(r \Omega) \partial_{r}\left(r^{2} \Omega\right)$. On the Rayleigh line, from (1) and (2), $\Omega(r)$ takes the simple form, $\Omega=b / r^{2}$, and so $\zeta=0$ at all $r$. We said earlier that in Fig. 2 the wavenumber changes little along the boundary, as its dependence is principally on the strength of the imposed field. The wavenumber parameter $\epsilon$ of Ji et al. (2001) then depends only on $Q$, so $\epsilon=\epsilon(Q)$. Ignoring factors that depend only on $\epsilon$ in the substitutions,

$S^{2}=P m Q, \quad R m=P m R e$,

(10) leads to the condition for stability,

$R e_{1} \leq \frac{A(Q)}{\sqrt{P m}}$

which confirms our result (9). This relation (12) should hold for all $P m$ as we have not been required to take any limits. The function $A(Q)$ has a minimum for some $Q$ and $\alpha(Q)$. For $\eta=0.5$ we found the minimum occurs at $Q=86, \alpha=2.1$.

Whilst the linear result (12) should hold for very small Pm, from the work of Wendt (1933) and Taylor (1936) a turbulent (nonlinear) instability is observed at large Reynolds numbers $\left(R e_{1} \gtrsim 2.5 \times 10^{5}\right)$ with this radius ratio. Extrapolating down one magnitude to $P m=10^{-5}$ 
for laboratory fluids gives $R e_{1 \mathrm{c}} \approx 2 \times 10^{5}$, a similar value, for the magneto-rotational (linear) instability.

A radial truncation of 12 Chebyshev modes was found sufficient for all calculations. In particular, for the calculation at $P m=10^{-4}$ in Fig. 4, convergence was tested by increasing the truncation and also decreasing the timestep. The fractional numerical error in $R e_{1 \mathrm{c}}$ is estimated at approximately $10^{-6}$.

\section{Discussion}

Our calculations show that many rotation laws of the form $\Omega(r)=a+b / r^{2}$ which are hydrodynamically stable (that is to say, they satisfy the Rayleigh criterion) become linearly unstable when a magnetic field is applied. Our results confirm the finding of Rüdiger \& Zhang (2001) and extend them in the Rayleigh stable region.

We have determined the instability at magnetic Prandtl numbers $P m$ one order of magnitude smaller than Rüdiger \& Zhang's, towards the small magnetic Prandtl number limit, which is relevant to possible MHD dynamo experiments with liquid sodium and gallium. Although the power law $R e_{1 \mathrm{c}} \propto P m^{-0.5}$ that we find on the Rayleigh line $\left(\mu=\eta^{2}\right)$ is slightly different from theirs $\left(R e_{1 \mathrm{c}} \propto P m^{-0.65}\right.$ on $\mu=\frac{1}{3}$ ), it confirms their conjecture that the nonlinear instability found by Richards \& Zahn (1999) and the MRI are likely to occur at Reynolds numbers of the same order of magnitude.

We also find that the flow becomes particularly unstable if the magnetic Prandtl number is greater than unity. The instability boundary in the $R e_{1}$ vs. $R e_{2}$ plane rapidly tends towards the solid body rotation line. This enhanced instability for large $P m$ is consistent with earlier results of Kurzweg (1963). His boundary conditions were selected such as to avoid mathematical difficulties but for small $\mathrm{Pm}$ agreed well with the results of Chandrasekhar (1961). The significance of the instability in this case is linked to the possibility (Kulsrud \& Anderson 1992; Brandenburg 2001) that large values of $P m$ exist in central regions of galaxies.

\section{References}

Balbus, S. A., \& Hawley, J. F. 1991, ApJ, 376, 214

Barenghi, C. F. 1991, J. Comput. Phys., 95, 175

Brandenburg, A. 2001, ApJ, 550, 824

Brandenburg, A., Nordlund, A., Stein, R. F., \& Torkelsson, U. 1995, ApJ, 446, 741

Chandrasekhar, S. 1961, Hydrodynamic and Hydromagnetic Stability (Oxford)

Drecker, A., Ruediger, R., \& Hollerbach, R. 2000, MNRAS, 317,45

Ji, H., Goodman, J., \& Kageyama, A. 2001, MNRAS, 325, L1 et al.

Jones, C. A. 1985, J. Fluid Mech., 157, 135

Kurzweg, U. H. 1963, J. Fluid Mech., 17, 52

Kulsrud, R. M., \& Anderson, S. W. 1992, ApJ, 396, 606

Marcus, P. S. 1984, J. Fluid Mech., 146, 45

Richards, D., \& Zahn, J. 1999, A\&A, 347, 734

Roberts, P. H. 1964, Proc. Cam. Phil. Soc., 60, 635

Rüdiger, G., \& Zhang, Y. 2001, A\&A, 378, 302

Sano, T., \& Miyama, S. 1999, ApJ, 515, 776

Taylor, G. I. 1936, Proc. Roy. Soc. London A, 157, 546

Tilgner, A. 2000, PEPI, 117, 171

Velikhov, E. P. 1959, Sov. Phys. JETP, 9, 995

Willis, A. P., \& Barenghi, C. F. 2002, J. Fluid Mech., in press

Wendt, F. 1933, Ing. Arch., 4, 577 